\title{
The transmissibility estimation of influenza with early stage data of small-scale outbreaks in Changsha, China, 2005-2013
}

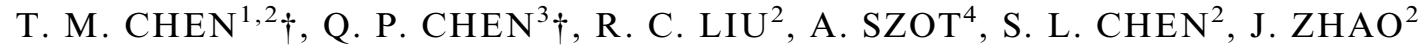 \\ AND S. S. ZHOU ${ }^{1 *}$ \\ ${ }^{1}$ Department of Malaria, National Institute of Parasitic Diseases, Chinese Center for Disease Control and \\ Prevention, Shanghai, People's Republic of China \\ ${ }^{2}$ Changsha Center for Disease Control and Prevention, Changsha, Hunan, People's Republic of China \\ ${ }^{3}$ Hospital, Shanghai Normal University, Shanghai, People's Republic of China \\ ${ }^{4}$ Mass Medical International, Brookline, MA, USA
}

Received 16 March 2016; Final revision 6 October 2016; Accepted 10 October 2016; first published online 11 November 2016

\section{SUMMARY}

Hundreds of small-scale influenza outbreaks in schools are reported in mainland China every year, leading to a heavy disease burden which seriously impacts the operation of affected schools. Knowing the transmissibility of each outbreak in the early stage has become a major concern for public health policy-makers and primary healthcare providers. In this study, we collected all the small-scale outbreaks in Changsha (a large city in south central China with $\sim 7.04$ million population) from January 2005 to December 2013. Four simple and popularly used models were employed to calculate the reproduction number $(R)$ of these outbreaks. Given that the duration of a generation interval $T c=2.7$ and the standard deviation (s.D.) $\sigma=1 \cdot 1$, the mean $R$ estimated by an epidemic model, normal distribution and delta distribution were $2 \cdot 51$ (S.D. $=0 \cdot 73$ ), $4 \cdot 11$ (s.D. $=2 \cdot 20$ ) and 5.88 (s.D. $=5.00$ ), respectively. When $T c=2.9$ and $\sigma=1.4$, the mean $R$ estimated by the three models were 2.62 (s.D. $=0.78), 4.72$ (s.D. $=2.82)$ and 6.86 (s.D. $=6.34)$, respectively. The mean $R$ estimated by gamma distribution was $4 \cdot 32$ (S.D. $=2 \cdot 47$ ). We found that the values of $R$ in small-scale outbreaks in schools were higher than in large-scale outbreaks in a neighbourhood, city or province. Normal distribution, delta distribution, and gamma distribution models seem to more easily overestimate the $R$ of influenza outbreaks compared to the epidemic model.

Key words: Influenza, mathematical model, reproduction number, small-scale outbreak.

\section{INTRODUCTION}

Each year, many countries or regions experience a peak influenza virus activity. A large number of people suffer

\footnotetext{
* Author for correspondence: Professor S. S. Zhou, Department of Malaria, National Institute of Parasitic Diseases, Chinese Center for Disease Control and Prevention, 207 Ruijin Er Road, Shanghai, People's Republic of China.

(Email: zss163@hotmail.com)

$\dagger$ These authors contributed equally to this work.
}

from the infection and many individuals are affected by small-scale outbreaks in schools. There are hundreds of such small-scale influenza outbreaks in mainland China every year, leading to a heavy disease burden which seriously impacts the operation of affected schools. Knowing the transmissibility of each outbreak in early stages has become a major concern for the public health policy-makers and primary healthcare providers. Thus, it is necessary to estimate the reproduction number $R$ (a most commonly used indicator that quantifies 
the transmissibility of influenza) when the primary health departments receive an outbreak report. However, the $R$ of small-scale outbreaks has not yet been well estimated in China.

$R$ is defined as the average number of secondary infections that arise from a typical primary case [1, 2]. From this definition, it is immediately clear that when $R>1$, the disease is able to spread in the susceptible population. If $R<1$, the infection will be cleared from the population. An individual-based model [3-5] or ordinary differential equation (ODE) model $[2,6]$ has been commonly employed for the estimation of $R$. In these models, the natural history of influenza (the incubation period, the latent period, infectious, or recovered, etc.), the demographic characteristics of the affected population, the epidemic data, and the countermeasures for controlling the outbreak are always included, which press the model closer to the actual scenarios hiding below the outbreak. Unfortunately, because of the professional gap between public health and the mathematical model, it is difficult for the primary health department in China to estimate the $R$ of an outbreak by using an individual-based model or an ODE model. It is imperative to confirm a practical and accurate transmissibility estimation method for the primary health department.

There are several simple approaches which could be used for the estimation of $R$ from epidemiological data. These are defined as 'susceptibles at endemic equilibrium', 'average age at infection', 'the final size equation', 'calculation from the intrinsic growth rate' by Heffernan et al. [7]. The epidemic model, normal distribution, delta distribution, and gamma distribution models by Wallinga et al. [8], Heffernan et al. [7] and Trichereau et al. [9] were also used. Heffernan's former three models are not easily used at the early stage of an outbreak because the parameters of these models are hard to obtain at that stage. The model 'calculation from the intrinsic growth rate' is the same as the epidemic model. Therefore, the epidemic model, along with the normal distribution, delta distribution, and gamma distribution models are the best choices for the model screening.

In this study, we collected all the small-scale outbreaks in Changsha from January 2005 to December 2013. The four simple and commonly used models [7-9] were employed to calculate the $R$ values of these outbreaks, and the results of each model were compared in order to commend an optimized model which could be used. We also collected the data of interventions employed in each outbreak and calculated the reproductive number with control measures $\left(R_{\text {con }}\right)$ of each outbreak to discover the effectiveness of the interventions.

\section{METHODS}

\section{Data collection}

In China, the criterion for an influenza outbreak has been defined as $\geqslant 10$ influenza-like illness (ILI) cases occurring in the same school, preschool, or other collective organization within 1 week, with laboratoryconfirmed influenza viruses through virus isolation or real-time reverse transcriptase-polymerase chain reaction analysis. Our study subjects also included public health incidents that were defined as $\geqslant 30$ ILIs within 1 week. ILI refers to a fever (axillary temperature $\geqslant 38^{\circ} \mathrm{C}$ ) accompanied by coughing or sore throat and a lack of a laboratory-confirmed diagnosis of the specific pathogen.

We built a dataset of seasonal influenza and influenza $\mathrm{A}(\mathrm{H} 1 \mathrm{~N} 1)$ pdm outbreaks by collecting information on all outbreaks reported from 1 January 2005 to 31 December 2013 in Changsha, China. Data included location type (e.g. primary school, middle school, high school, and prison), the school population, the date of the reported outbreak, the date of symptom onset of all cases, the subtype of influenza virus, and interventions including symptom surveillance, case isolation, symptomatic treatment of cases, antivirals for treatment or prophylaxis use, health education, environment disinfection, vaccination, social distance, and class, grade and school closure.

Symptom surveillance which focused on ILI cases was implemented every day from the reported outbreak date to the end of the outbreak. For case isolation, infected individuals were isolated in the hospital if they reported severe symptoms like pneumonia, or were isolated at home for mild cases until all the symptoms disappeared after $24 \mathrm{~h}$ or $48 \mathrm{~h}$. For symptomatic treatment, cases were treated by medication (not antivirals) to relieve the symptoms in the hospital or at home.

Local CDC staff had overseen health education for the affected people who were taught to maintain personal hygiene and wear a gauze mask during the outbreak. Local public health providers also disinfected the potential environment or air contaminated by cases, and a chlorine-based disinfectant was employed to sterilize the fomites and a peracetic acid solution of $15 \%$ concentration was employed to disinfect the air through fumigation. 
During the period of the class/grade/school closure, a teacher in charge of a class was required to monitor all the students in the class every day. At the same time, each student was asked to stay at home, take their own temperature and report their findings to their teacher by telephone.

Two medications (moroxydine and a traditional medicine called 'Ban Lan Gen Chong Ji') were used for prophylaxis. Moroxydine was prescribed at $0 \cdot 1 \mathrm{~g}$ t.i.d. for 2 or 3 days for children aged $<10$ years and the dose was doubled for those aged $>10$ years. 'Ban Lan Gen Chong Ji', which is made from an herbaceous plant named Ban Lan Gen, was used at $10 \mathrm{~g}$ t.i.d. for 5 days.

In our study, 56 influenza outbreaks in school, prison and the community were collected (Table 1). To make the estimation of $R$ more stable and reliable, the inclusion criterion was that the exponential growth phase of outbreak should be $\geqslant 3$ days. The exclusion criteria were as follows: $(a)$ that the outbreaks had no illness onset date of each case, or only a part of the cases had an onset date, which may make the epidemic curve of the outbreak unavailable for the estimation of $R$; (b) that there was no influenza laboratory test results in the outbreak; $(c)$ there was a combined infection of influenza subtypes/types in an outbreak; and (d) the virus was untyped. According to these criteria only the target datasets would be included in our study for estimating the $R$ of each outbreak.

\section{Estimation of reproduction number}

To estimate the $R$ of each outbreak, we employed the intrinsic growth rate method using the following four models [7-9].

\section{Epidemic model}

In this model, the reproduction number, $R$, is expressed as follows:

$R=1+r T c$.

In this equation, $r$ and $T c$ refer to the epidemic growth rate and the duration of a generation interval.

\section{Normal distribution}

In this model, the generation intervals may approximate a normal distribution, making $R$ as follows:

$R=\mathrm{e}^{r T c-(1 / 2) r^{2} \sigma^{2}}$ where $T c$ refers to the mean generation interval and $\sigma$ refers to the standard deviation (s.D.).

\section{Delta distribution}

In this model, all secondary infections are exactly equal to the mean generation interval $T c$. The model is expressed as follows:

$R=e^{r T c}$.

\section{Gamma distribution}

For the gamma distribution $\operatorname{Gamma}(\alpha, \beta)$, the relationship between $r$ and $R$ is expressed as follows:

$R=(1+\beta r)^{\alpha}$.

During the exponential growth phase of an outbreak, the relationship between the growth rate $r$ and daily incidence $I(t)$ of symptom onset can be expressed as $\mathrm{d} I / \mathrm{d} t=$ $r I$, where $r$ is the exponential growth rate [7, 10]. The exponential growth rate $r$ was estimated from the daily epidemic curve of each outbreak we collected.

As $T c$ could not be estimated from our epidemic data, we used two different generation intervals derived from previous research $[10,11]$. The mean generation intervals and standard deviations were $T c=2 \cdot 7, \sigma=1 \cdot 1$ [11] and $T c=2 \cdot 9, \sigma=1 \cdot 4$ [10]. The two parameters $\alpha$ and $\beta$ of a gamma distribution Gamma $(\alpha, \beta)$ were $4 \cdot 2$ and $0 \cdot 68$, respectively [10]. The values of parameters $(T c=2.9, \sigma=1.4)$ and Gamma $(4 \cdot 2,0 \cdot 68)$ were calculated from the same data [10].

\section{Simulation methods}

Berkeley Madonna v. 8.3.18 (University of California at Berkeley, USA) and Microsoft Office Excel 2003 (Microsoft, USA) software were employed for model simulation and figure development, respectively. The details of model-fitting methods run in Berkeley Madonna, such as the Runge-Kutta method of order 4 and root-mean-square deviation, were the same as the those in references [1, 2, 12].

\section{Ethical standards}

The authors assert that all procedures contributing to this work comply with the ethical standards of the relevant national and institutional committees on human experimentation and with the Helsinki Declaration of 1975, as revised in 2008. 
Table 1. General information of 56 influenza outbreaks in Changsha city, China, 2005-2013 ( $n=56$ )

\begin{tabular}{|c|c|c|c|c|c|c|c|}
\hline Outbreak ID & Year & Month & Type of locations & Population & Accumulative cases & DO & Subtypes \\
\hline 1 & 2009 & 10 & Secondary school & 1101 & 44 & 14 & H1N1pdm \\
\hline 2 & 2009 & 11 & Secondary school & 4644 & 143 & 11 & H1N1pdm \\
\hline 3 & 2009 & 11 & Secondary school & 1811 & 77 & 12 & H1N1pdm \\
\hline 4 & 2009 & 11 & Primary school & 1231 & 71 & 11 & H1N1pdm \\
\hline 5 & 2009 & 11 & Primary school & 1028 & 58 & 21 & H1N1pdm \\
\hline 6 & 2009 & 11 & Secondary school & 1874 & 38 & 10 & H1N1pdm \\
\hline 7 & 2009 & 11 & Secondary school & 1192 & 59 & 15 & H1N1pdm \\
\hline 8 & 2009 & 11 & Secondary school & 1342 & 256 & 40 & H1N1pdm \\
\hline 9 & 2009 & 11 & College & 1357 & 61 & 23 & H1N1pdm \\
\hline 10 & 2009 & 10 & Training school & 126 & 58 & 9 & H1N1pdm \\
\hline 11 & 2009 & 10 & Primary school & 1129 & 107 & 14 & H1N1pdm \\
\hline 12 & 2009 & 9 & College & 6546 & 114 & 22 & H1N1pdm \\
\hline 13 & 2009 & 10 & College & 13485 & 163 & 9 & H1N1pdm \\
\hline 14 & 2009 & 11 & Prison & 694 & 83 & 17 & H1N1pdm \\
\hline 15 & 2009 & 11 & Primary school & 264 & 66 & 11 & H1N1pdm \\
\hline 16 & 2009 & 11 & Secondary school & 1240 & 100 & 11 & H1N1pdm \\
\hline 17 & 2009 & 11 & Secondary school & 2050 & 101 & 14 & H1N1pdm \\
\hline 18 & 2009 & 11 & Secondary school & 1138 & 93 & 15 & H1N1pdm \\
\hline 19 & 2009 & 11 & Primary school & 1563 & 42 & 17 & H1N1pdm \\
\hline 20 & 2009 & 11 & Secondary school & 4673 & 17 & 13 & H1N1pdm \\
\hline 21 & 2009 & 11 & Secondary school & 1950 & 18 & 4 & H1N1pdm \\
\hline 22 & 2009 & 10 & Secondary school & 4290 & 31 & 10 & H1N1pdm \\
\hline 23 & 2009 & 10 & Secondary school & 2670 & 95 & 17 & H1N1pdm \\
\hline 24 & 2009 & 9 & Secondary school & 4297 & 89 & 19 & H1N1pdm \\
\hline 25 & 2009 & 10 & College & 2477 & 43 & 7 & H1N1pdm \\
\hline 26 & 2009 & 9 & College & 1434 & 48 & 13 & H1N1pdm \\
\hline 27 & 2009 & 11 & Primary school & 1081 & 155 & 21 & H1N1pdm \\
\hline 28 & 2009 & 10 & Secondary school & 588 & 37 & 15 & H1N1pdm \\
\hline 29 & 2009 & 11 & Primary school & 429 & 19 & 10 & H1N1pdm \\
\hline 30 & 2009 & 11 & Secondary school & 2280 & 96 & 43 & H1N1pdm \\
\hline 31 & 2009 & 11 & Secondary school & 1251 & 127 & 20 & H1N1pdm \\
\hline 32 & 2009 & 11 & Secondary school & 1562 & 121 & 25 & H1N1pdm \\
\hline 33 & 2009 & 11 & Secondary school & 5313 & 11 & 21 & H1N1pdm \\
\hline 34 & 2009 & 11 & Secondary school & 2396 & 791 & 9 & H1N1pdm \\
\hline 35 & 2010 & 1 & Prison & 628 & 86 & 12 & H1N1pdm \\
\hline 36 & 2009 & 9 & Secondary school & 4032 & 60 & 15 & H1N1pdm \\
\hline 37 & 2009 & 11 & College & 2255 & 49 & 15 & H1N1pdm \\
\hline 38 & 2013 & 1 & Secondary school & 2500 & 80 & 19 & H1N1pdm \\
\hline 39 & 2013 & 11 & Secondary school & 1490 & 44 & 15 & $\mathrm{H} 3 \mathrm{~N} 2$ \\
\hline 40 & 2013 & 11 & Secondary school & 4872 & 74 & 17 & $\mathrm{H} 3 \mathrm{~N} 2+\mathrm{B}$ \\
\hline 41 & 2009 & 8 & Community & 1900 & 41 & 10 & $\mathrm{H} 3 \mathrm{~N} 2$ \\
\hline 42 & 2009 & 2 & Secondary school & 7716 & 336 & 23 & H1N1 \\
\hline 43 & 2009 & 3 & Primary school & 671 & 43 & 15 & B \\
\hline 44 & 2009 & 3 & Primary school & 885 & 43 & 13 & $\mathrm{H} 1 \mathrm{~N} 1+\mathrm{B}$ \\
\hline 45 & 2009 & 3 & Secondary school & 639 & 39 & 8 & H1N1 \\
\hline 46 & 2009 & 3 & Secondary school & 855 & 32 & 7 & H1N1 \\
\hline 47 & 2008 & 2 & Primary school & 160 & 28 & 9 & $\mathrm{H} 3 \mathrm{~N} 2$ \\
\hline 48 & 2008 & 6 & Primary school & 125 & 51 & 20 & B \\
\hline 49 & 2007 & 3 & Primary school & 375 & 58 & 15 & A (untyped) \\
\hline 50 & 2007 & 3 & Secondary school & 1539 & 42 & 9 & A (untyped) \\
\hline 51 & 2006 & 4 & Primary school & 110 & 47 & n.a. & Untyped \\
\hline 52 & 2006 & 2 & Primary school & 570 & 96 & 11 & A (untyped) \\
\hline 53 & 2006 & 2 & Primary school & 187 & 58 & 10 & A (untyped) \\
\hline 54 & 2006 & 2 & Secondary school & 210 & 47 & 13 & A (untyped) \\
\hline
\end{tabular}


Table 1 (cont.)

\begin{tabular}{llllllll}
\hline \hline Outbreak ID & Year & Month & Type of locations & Population & Accumulative cases & DO & Subtypes \\
\hline 55 & 2005 & 12 & Secondary school & 1043 & 49 & 9 & A (untyped) \\
56 & 2005 & 3 & Primary school & 317 & 27 & n.a. & n.a. \\
\hline \hline
\end{tabular}

ID, Identification; DO, duration of outbreak; n.a., not available.

Table 2. The growth rate $r$ of 32 influenza outbreaks in Changsha city, China, 2005-2013 ( $n=32)$

\begin{tabular}{|c|c|c|c|c|}
\hline \multirow[b]{2}{*}{ Outbreak ID } & \multicolumn{2}{|l|}{$r$} & \multirow[b]{2}{*}{$R^{2}$} & \multirow[b]{2}{*}{$P$ value } \\
\hline & Value & $95 \% \mathrm{CI}$ & & \\
\hline 1 & 0.693 & $0 \cdot 565-0 \cdot 821$ & 0.936 & $0 \cdot 033$ \\
\hline 3 & $0 \cdot 876$ & $0 \cdot 370-1 \cdot 382$ & $0 \cdot 428$ & $0 \cdot 159$ \\
\hline 5 & $0 \cdot 351$ & $0 \cdot 020-0.682$ & $0 \cdot 158$ & $0 \cdot 330$ \\
\hline 6 & $1 \cdot 040$ & $0 \cdot 840-1 \cdot 240$ & 0.964 & $0 \cdot 121$ \\
\hline 7 & $0 \cdot 746$ & $0 \cdot 374-1 \cdot 118$ & $0 \cdot 446$ & $0 \cdot 101$ \\
\hline 8 & $0 \cdot 475$ & $0.415-0.535$ & $0 \cdot 898$ & $0 \cdot 000$ \\
\hline 9 & $0 \cdot 585$ & $0 \cdot 477-0.693$ & $0 \cdot 879$ & $0 \cdot 006$ \\
\hline 10 & $0 \cdot 492$ & $0 \cdot 419-0.565$ & 0.919 & $0 \cdot 003$ \\
\hline 11 & $1 \cdot 287$ & $0 \cdot 487-2 \cdot 087$ & $0 \cdot 463$ & $0 \cdot 206$ \\
\hline 13 & $0 \cdot 331$ & $0 \cdot 190-0 \cdot 472$ & 0.477 & $0 \cdot 058$ \\
\hline 14 & $0 \cdot 707$ & $0 \cdot 611-0 \cdot 803$ & 0.965 & $0 \cdot 018$ \\
\hline 17 & $0 \cdot 743$ & $0 \cdot 595-0.891$ & $0 \cdot 894$ & $0 \cdot 015$ \\
\hline 18 & $0 \cdot 812$ & $0 \cdot 298-1 \cdot 326$ & $0 \cdot 333$ & $0 \cdot 175$ \\
\hline 19 & $0 \cdot 601$ & $0 \cdot 293-0.909$ & $0 \cdot 322$ & $0 \cdot 087$ \\
\hline 21 & $1 \cdot 151$ & $1 \cdot 121-1 \cdot 181$ & 0.999 & $0 \cdot 017$ \\
\hline 22 & $0 \cdot 786$ & $0 \cdot 278-1 \cdot 294$ & $0 \cdot 374$ & $0 \cdot 197$ \\
\hline 25 & $0 \cdot 279$ & $0 \cdot 042-0 \cdot 516$ & $0 \cdot 316$ & $0 \cdot 324$ \\
\hline 26 & $0 \cdot 484$ & $0 \cdot 267-0 \cdot 701$ & $0 \cdot 416$ & $0 \cdot 061$ \\
\hline 27 & $0 \cdot 786$ & $0.694-0.878$ & 0.948 & $0 \cdot 001$ \\
\hline 28 & $1 \cdot 040$ & $0 \cdot 517-1 \cdot 563$ & 0.798 & $0 \cdot 297$ \\
\hline 31 & $0 \cdot 769$ & $0.631-0.907$ & 0.912 & $0 \cdot 011$ \\
\hline 35 & $0 \cdot 870$ & $0 \cdot 569-1 \cdot 171$ & $0 \cdot 806$ & $0 \cdot 102$ \\
\hline 36 & $0 \cdot 389$ & $0 \cdot 212-0 \cdot 566$ & 0.617 & $0 \cdot 115$ \\
\hline 37 & $0 \cdot 769$ & $0 \cdot 344-1 \cdot 194$ & $0 \cdot 396$ & $0 \cdot 130$ \\
\hline 38 & $0 \cdot 179$ & $0 \cdot 119-0 \cdot 239$ & $0 \cdot 524$ & $0 \cdot 018$ \\
\hline 39 & $0 \cdot 168$ & $0 \cdot 160-0 \cdot 176$ & 0.998 & $0 \cdot 031$ \\
\hline 42 & $0 \cdot 966$ & $0 \cdot 858-1 \cdot 074$ & 0.975 & $0 \cdot 012$ \\
\hline 43 & $0 \cdot 415$ & $0 \cdot 300-0 \cdot 530$ & $0 \cdot 764$ & $0 \cdot 023$ \\
\hline 45 & 0.638 & $0 \cdot 458-0 \cdot 818$ & $0 \cdot 806$ & $0 \cdot 039$ \\
\hline 46 & $0 \cdot 559$ & $0 \cdot 466-0 \cdot 652$ & 0.922 & $0 \cdot 009$ \\
\hline 47 & $1 \cdot 151$ & $0 \cdot 781-1 \cdot 521$ & 0.906 & $0 \cdot 198$ \\
\hline 48 & $0 \cdot 564$ & $0 \cdot 285-0 \cdot 843$ & $0 \cdot 368$ & $0 \cdot 083$ \\
\hline
\end{tabular}

ID, Identification; $r$, epidemic growth rate; CI, confidence interval; $R^{2}$, coefficient of determination.

\section{RESULTS}

After analysing 56 outbreaks, 32 outbreaks were enrolled for estimating $R$, because three outbreaks had no illness onset dates for each case and one had no laboratory test results. Moreover, the data of four outbreaks were not integrated, the combined infection of influenza subtypes/types were tested in two outbreaks, six outbreaks were untyped, and the exponential growth phase of nine outbreaks were $<3$ days. Of these 32 outbreaks, the growth rates of 15 were shown to have statistical significance by curve fitting (see Table 2). According to the generation interval used and its distribution, $R$ estimations are shown in Table 3 .

Given that $T c=2.7$ and $\sigma=1 \cdot 1$, the mean $R$ estimated by the epidemic model, normal distribution and delta distribution models were 2.68 (s.D. $=0.71$ ), 4.58 (S.D. $=2 \cdot 22$ ) and $6 \cdot 77$ (S.D. $=5 \cdot 28$ ), respectively. Given that $T c=2 \cdot 9$ and $\sigma=1 \cdot 4$, the mean $R$ estimated by the three models were $2 \cdot 80$ (s.D. $=0 \cdot 76$ ), $5 \cdot 31$ (s.D. $=$ $2 \cdot 87$ ) and 7.95 (s.D. $=6.73$ ), respectively. The mean $R$ estimated by gamma distribution was 4.84 (S.D. $=$ $2 \cdot 52$ ) (see Table 3). The mean values of $R$ estimated by normal distribution were higher than the those estimated by the epidemic model, even if $T c=2 \cdot 7(t=-$ $3.149, P=0.006)$ or $T c=2 \cdot 9 \quad(t=3.265, P=0.005)$. Similarly, the mean values of $R$ estimated by delta distribution were higher than those estimated by the epidemic model, even if $T c=2 \cdot 7(t=-2.974, P=0.010)$ or $T c=2.9(t=-2.939, P=0.011)$. However, there was no significance between the mean values of $R$ estimated by delta distribution and those estimated by normal distribution, even if $T c=2.7(t=-1.483, P=$ $0 \cdot 149)$ or $T c=2 \cdot 9(t=-1 \cdot 396, P=0 \cdot 174)$.

Because $T c=2 \cdot 9, \sigma=1.4$ and Gamma $(4 \cdot 2,0.68)$ were from the same data, we only compared the results of the gamma distribution estimated with the results of the epidemic model, normal distribution and delta distribution models that had been tested under the same conditions. The mean $R$ estimated by gamma distribution was higher than the mean $R$ estimated by the epidemic model $(t=-2.988, P=0 \cdot 008)$. However, the mean $R$ estimated by gamma distribution was not significant compared to the mean $R$ estimated by normal distribution $(t=0.478, P=0.636)$ and delta distribution $(t=1 \cdot 676, P=0 \cdot 105)$.

Of the 15 outbreaks, ten were H1N1pdm, one was $\mathrm{H} 3 \mathrm{~N} 2$, three were $\mathrm{H} 1 \mathrm{~N} 1$ and one was B subtype. 
Table 3. The reproduction number $R$ of 15 influenza outbreaks in Changsha city, China, 2005-2013 ( $n=15)$

\begin{tabular}{|c|c|c|c|c|c|c|c|c|c|c|c|c|c|c|}
\hline \multirow[b]{2}{*}{ Outbreak ID } & \multicolumn{2}{|c|}{$\begin{array}{l}\text { Epidemic model } \\
(T c=2 \cdot 7)\end{array}$} & \multicolumn{2}{|c|}{$\begin{array}{l}\text { Normal } \\
\text { distribution }(T c= \\
2 \cdot 7, \sigma=1 \cdot 1)\end{array}$} & \multicolumn{2}{|c|}{$\begin{array}{l}\text { Delta distribution } \\
(T c=2 \cdot 7)\end{array}$} & \multicolumn{2}{|c|}{$\begin{array}{l}\text { Epidemic model } \\
(T c=2 \cdot 9)\end{array}$} & \multicolumn{2}{|c|}{$\begin{array}{l}\text { Normal distribution } \\
(T c=2 \cdot 9, \sigma=1 \cdot 4)\end{array}$} & \multicolumn{2}{|c|}{$\begin{array}{l}\text { Delta distribution } \\
(T c=2 \cdot 9)\end{array}$} & \multicolumn{2}{|c|}{$\begin{array}{l}\text { Gamma distribution } \\
\operatorname{Gamma}(4 \cdot 2,0 \cdot 68)\end{array}$} \\
\hline & $R$ & $95 \% \mathrm{CI}$ & $R$ & $95 \% \mathrm{CI}$ & $R$ & $95 \% \mathrm{CI}$ & $R$ & $95 \% \mathrm{CI}$ & $R$ & $95 \% \mathrm{CI}$ & $R$ & $95 \% \mathrm{CI}$ & $R$ & $95 \% \mathrm{CI}$ \\
\hline 1 & $2 \cdot 87$ & $2 \cdot 53-3 \cdot 22$ & $4 \cdot 86$ & $3 \cdot 79-6 \cdot 10$ & $6 \cdot 50$ & $4 \cdot 60-9 \cdot 18$ & $3 \cdot 01$ & $2 \cdot 64-3 \cdot 38$ & $5 \cdot 58$ & $4 \cdot 24-7 \cdot 19$ & $7 \cdot 46$ & $5 \cdot 15-10 \cdot 81$ & $5 \cdot 06$ & $3 \cdot 92-6 \cdot 44$ \\
\hline 8 & $2 \cdot 28$ & $2 \cdot 12-2 \cdot 44$ & $3 \cdot 15$ & $2 \cdot 76-3 \cdot 57$ & $3 \cdot 61$ & $3 \cdot 07-4 \cdot 24$ & $2 \cdot 38$ & $2 \cdot 20-2 \cdot 55$ & $3 \cdot 46$ & $3 \cdot 00-3 \cdot 97$ & $3 \cdot 96$ & $3 \cdot 33-4 \cdot 72$ & $3 \cdot 24$ & $2 \cdot 84-3 \cdot 68$ \\
\hline 9 & $2 \cdot 58$ & $2 \cdot 29-2 \cdot 87$ & $3 \cdot 95$ & $3 \cdot 16-4 \cdot 86$ & $4 \cdot 85$ & $3 \cdot 63-6 \cdot 50$ & $2 \cdot 70$ & $2 \cdot 38-3 \cdot 01$ & $4 \cdot 43$ & $3 \cdot 48-5 \cdot 58$ & $5 \cdot 45$ & $3 \cdot 99-7 \cdot 46$ & $4 \cdot 08$ & $3 \cdot 25-5 \cdot 06$ \\
\hline 10 & $2 \cdot 33$ & $2 \cdot 13-2 \cdot 53$ & $3 \cdot 26$ & $2 \cdot 79-3 \cdot 79$ & $3 \cdot 77$ & $3 \cdot 10-4 \cdot 60$ & $2 \cdot 43$ & $2 \cdot 22-2 \cdot 64$ & $3 \cdot 60$ & $3 \cdot 03-4 \cdot 24$ & $4 \cdot 17$ & $3 \cdot 37-5 \cdot 15$ & $3 \cdot 36$ & $2 \cdot 87-3 \cdot 92$ \\
\hline 14 & $2 \cdot 91$ & $2 \cdot 65-3 \cdot 17$ & 4.99 & $4 \cdot 15-5 \cdot 92$ & $6 \cdot 75$ & $5 \cdot 21-8 \cdot 74$ & $3 \cdot 05$ & $2 \cdot 77-3 \cdot 33$ & $5 \cdot 74$ & $4 \cdot 69-6 \cdot 95$ & $7 \cdot 77$ & $5 \cdot 88-10 \cdot 26$ & $5 \cdot 20$ & $4 \cdot 30-6 \cdot 23$ \\
\hline 17 & $3 \cdot 01$ & $2 \cdot 61-3 \cdot 41$ & $5 \cdot 32$ & $4 \cdot 02-6 \cdot 86$ & $7 \cdot 43$ & 4.99-11.09 & $3 \cdot 15$ & $2 \cdot 73-3 \cdot 58$ & $6 \cdot 18$ & $4 \cdot 53-8 \cdot 20$ & $8 \cdot 63$ & $5 \cdot 62-13 \cdot 25$ & $5 \cdot 57$ & $4 \cdot 17-7 \cdot 31$ \\
\hline 21 & $4 \cdot 11$ & $4 \cdot 03-4 \cdot 19$ & $10 \cdot 04$ & $9 \cdot 64-10 \cdot 43$ & $22 \cdot 37$ & $20 \cdot 63-24 \cdot 26$ & $4 \cdot 34$ & $4 \cdot 25-4 \cdot 42$ & $12 \cdot 63$ & $12 \cdot 07-13 \cdot 21$ & $28 \cdot 16$ & $25 \cdot 81-30 \cdot 72$ & $11 \cdot 34$ & $10 \cdot 80-11 \cdot 89$ \\
\hline 27 & $3 \cdot 12$ & $2 \cdot 87-3 \cdot 37$ & $5 \cdot 75$ & $4 \cdot 87-6 \cdot 71$ & $8 \cdot 35$ & $6 \cdot 51-10 \cdot 70$ & $3 \cdot 28$ & $3 \cdot 01-3 \cdot 55$ & $6 \cdot 72$ & $5 \cdot 59-8 \cdot 00$ & $9 \cdot 77$ & $7 \cdot 48-12 \cdot 76$ & $6 \cdot 04$ & $5 \cdot 07-7 \cdot 14$ \\
\hline 31 & $3 \cdot 08$ & $2 \cdot 70-3 \cdot 45$ & $5 \cdot 58$ & $4 \cdot 32-7 \cdot 04$ & $7 \cdot 97$ & $5 \cdot 49-11 \cdot 58$ & $3 \cdot 23$ & $2 \cdot 83-3 \cdot 63$ & $6 \cdot 50$ & $4 \cdot 90-8 \cdot 44$ & $9 \cdot 30$ & $6 \cdot 23-13 \cdot 88$ & $5 \cdot 85$ & $4 \cdot 48-7 \cdot 52$ \\
\hline 38 & $1 \cdot 48$ & $1 \cdot 32-1 \cdot 65$ & $1 \cdot 59$ & $1 \cdot 37-1 \cdot 84$ & $1 \cdot 62$ & $1 \cdot 38-1 \cdot 91$ & $1 \cdot 52$ & $1 \cdot 35-1 \cdot 69$ & $1 \cdot 65$ & $1 \cdot 40-1 \cdot 93$ & $1 \cdot 68$ & $1 \cdot 41-2 \cdot 00$ & $1 \cdot 62$ & $1 \cdot 39-1 \cdot 88$ \\
\hline 39 & $1 \cdot 45$ & $1 \cdot 43-1 \cdot 48$ & $1 \cdot 55$ & $1 \cdot 52-1 \cdot 58$ & $1 \cdot 57$ & $1 \cdot 54-1 \cdot 61$ & $1 \cdot 49$ & $1 \cdot 46-1 \cdot 51$ & $1 \cdot 60$ & $1 \cdot 57-1 \cdot 64$ & $1 \cdot 63$ & $1 \cdot 59-1 \cdot 67$ & $1 \cdot 58$ & $1 \cdot 54-1 \cdot 61$ \\
\hline 42 & $3 \cdot 61$ & $3 \cdot 32-3 \cdot 90$ & $7 \cdot 72$ & $6 \cdot 50-9 \cdot 04$ & $13 \cdot 57$ & $10 \cdot 14-18 \cdot 17$ & $3 \cdot 80$ & $3 \cdot 49-4 \cdot 11$ & $9 \cdot 36$ & $7 \cdot 71-11 \cdot 21$ & $16 \cdot 47$ & $12 \cdot 04-22 \cdot 52$ & $8 \cdot 34$ & $6 \cdot 89-10 \cdot 00$ \\
\hline 43 & $2 \cdot 12$ & $1 \cdot 81-2 \cdot 43$ & $2 \cdot 76$ & $2 \cdot 13-3 \cdot 53$ & $3 \cdot 07$ & $2 \cdot 25-4 \cdot 18$ & $2 \cdot 20$ & $1 \cdot 87-2 \cdot 54$ & $3 \cdot 00$ & $2 \cdot 26-3 \cdot 92$ & $3 \cdot 33$ & $2 \cdot 39-4 \cdot 65$ & $2 \cdot 84$ & $2 \cdot 18-3 \cdot 64$ \\
\hline 45 & $2 \cdot 72$ & $2 \cdot 24-3 \cdot 21$ & $4 \cdot 38$ & $3 \cdot 03-6 \cdot 07$ & $5 \cdot 60$ & $3 \cdot 44-9 \cdot 10$ & $2 \cdot 85$ & $2 \cdot 33-3 \cdot 37$ & $4 \cdot 97$ & $3 \cdot 32-7 \cdot 15$ & $6 \cdot 36$ & $3 \cdot 77-10 \cdot 72$ & $4 \cdot 54$ & $3 \cdot 12-6 \cdot 41$ \\
\hline 46 & $2 \cdot 51$ & $2 \cdot 26-2 \cdot 76$ & $3 \cdot 74$ & $3 \cdot 09-4 \cdot 50$ & $4 \cdot 52$ & $3 \cdot 52-5 \cdot 81$ & $2 \cdot 62$ & $2 \cdot 35-2 \cdot 89$ & $4 \cdot 19$ & $3 \cdot 39-5 \cdot 12$ & $5 \cdot 06$ & $3 \cdot 86-6 \cdot 62$ & $3 \cdot 87$ & $3 \cdot 18-4 \cdot 67$ \\
\hline
\end{tabular}

ID, Identification; $T c$, mean generation interval; $\sigma$, standard deviation; $R$, reproduction number; CI, confidence interval. 
Table 4. The results of ANOVA analysis in different subtypes of influenza virus ( $n=15)$

\begin{tabular}{|c|c|c|c|c|c|c|}
\hline Models & Subtypes & No. of outbreaks & Mean of $R$ & $95 \% \mathrm{CI}$ & $F$ value & $P$ value \\
\hline \multirow{5}{*}{ Epidemic $(T c=2 \cdot 7)$} & H1N1pdm & 10 & $2 \cdot 78$ & $2 \cdot 29-3 \cdot 27$ & \multirow{5}{*}{$1 \cdot 591$} & \multirow{5}{*}{$0 \cdot 247$} \\
\hline & $\mathrm{H} 3 \mathrm{~N} 2$ & 1 & $1 \cdot 45$ & - & & \\
\hline & $\mathrm{H} 1 \mathrm{~N} 1$ & 3 & $2 \cdot 95$ & $1 \cdot 50-4 \cdot 40$ & & \\
\hline & B & 1 & $2 \cdot 12$ & - & & \\
\hline & Total & 15 & $2 \cdot 68$ & $2 \cdot 29-3 \cdot 07$ & & \\
\hline \multirow[t]{5}{*}{ Normal distribution $(T c=2 \cdot 7, \sigma=1 \cdot 1)$} & H1N1pdm & 10 & $4 \cdot 85$ & $3 \cdot 24-6 \cdot 46$ & \multirow{5}{*}{0.988} & \multirow{5}{*}{$0 \cdot 434$} \\
\hline & $\mathrm{H} 3 \mathrm{~N} 2$ & 1 & 1.55 & - & & \\
\hline & $\mathrm{H} 1 \mathrm{~N} 1$ & 3 & $5 \cdot 28$ & $0-10.59$ & & \\
\hline & B & 1 & $2 \cdot 76$ & - & & \\
\hline & Total & 15 & $4 \cdot 58$ & $3 \cdot 34-5 \cdot 81$ & & \\
\hline \multirow{5}{*}{ Delta distribution $(T c=2 \cdot 7)$} & H1N1pdm & 10 & $7 \cdot 32$ & $3 \cdot 23-11 \cdot 41$ & \multirow{5}{*}{$0 \cdot 509$} & \multirow{5}{*}{$0 \cdot 684$} \\
\hline & $\mathrm{H} 3 \mathrm{~N} 2$ & 1 & $1 \cdot 57$ & - & & \\
\hline & H1N1 & 3 & $7 \cdot 90$ & $0-20 \cdot 18$ & & \\
\hline & B & 1 & $3 \cdot 07$ & - & & \\
\hline & Total & 15 & $6 \cdot 77$ & $3 \cdot 85-9 \cdot 69$ & & \\
\hline \multirow[t]{5}{*}{ Epidemic $(T c=2 \cdot 9)$} & H1N1pdm & 10 & $2 \cdot 91$ & $2 \cdot 38-3 \cdot 43$ & \multirow{5}{*}{1.590} & \multirow{5}{*}{$0 \cdot 248$} \\
\hline & $\mathrm{H} 3 \mathrm{~N} 2$ & 1 & $1 \cdot 49$ & - & & \\
\hline & H1N1 & 3 & 3.09 & $1 \cdot 54-4 \cdot 64$ & & \\
\hline & B & 1 & $2 \cdot 20$ & - & & \\
\hline & Total & 15 & $2 \cdot 80$ & $2 \cdot 38-3 \cdot 22$ & & \\
\hline \multirow{5}{*}{ Normal distribution $(T c=2 \cdot 9, \sigma=1 \cdot 4)$} & H1N1pdm & 10 & $5 \cdot 65$ & $3 \cdot 55-7 \cdot 75$ & \multirow{5}{*}{$0 \cdot 887$} & \multirow{5}{*}{$0 \cdot 478$} \\
\hline & $\mathrm{H} 3 \mathrm{~N} 2$ & 1 & $1 \cdot 60$ & - & & \\
\hline & H1N1 & 3 & $6 \cdot 17$ & $0-13 \cdot 10$ & & \\
\hline & B & 1 & $3 \cdot 00$ & - & & \\
\hline & Total & 15 & $5 \cdot 31$ & $3 \cdot 72-6 \cdot 90$ & & \\
\hline \multirow{5}{*}{ Delta distribution $(T c=2 \cdot 9)$} & H1N1pdm & 10 & $8 \cdot 64$ & $3 \cdot 38-13 \cdot 89$ & \multirow{5}{*}{$0 \cdot 465$} & \multirow{5}{*}{$0 \cdot 713$} \\
\hline & H3N2 & 1 & $1 \cdot 63$ & - & & \\
\hline & H1N1 & 3 & $9 \cdot 30$ & $0-24 \cdot 81$ & & \\
\hline & B & 1 & $3 \cdot 33$ & - & & \\
\hline & Total & 15 & $7 \cdot 95$ & $4 \cdot 22-11 \cdot 68$ & & \\
\hline \multirow[t]{5}{*}{ Gamma distribution Gamma $(4 \cdot 2,0 \cdot 68)$} & H1N1pdm & 10 & $5 \cdot 14$ & $3 \cdot 29-6 \cdot 99$ & \multirow{5}{*}{$0 \cdot 876$} & \multirow{5}{*}{$0 \cdot 483$} \\
\hline & $\mathrm{H} 3 \mathrm{~N} 2$ & 1 & 1.58 & - & & \\
\hline & $\mathrm{H} 1 \mathrm{~N} 1$ & 3 & $5 \cdot 58$ & $0-11.57$ & & \\
\hline & B & 1 & $2 \cdot 84$ & - & & \\
\hline & Total & 15 & $4 \cdot 84$ & $3 \cdot 44-6 \cdot 23$ & & \\
\hline
\end{tabular}

$T \mathrm{c}$, Mean generation interval; $\sigma$, standard deviation; $R$, reproduction number; $\mathrm{CI}$, confidence interval; $F$, a statistic value in analysis of variance.

There was no significance with the mean $R$ of these subtypes based on the analysis of variance (ANOVA), even if $R$ was estimated by the four models under different conditions (see Table 4).

In the 15 outbreaks used for calculating $R$, we found that eight interventions, implemented individually or in combination, were employed after the reported outbreak date, except with social distance and antivirals like oseltamivir (see Table 5). These interventions included five non-pharmaceutical (symptom surveillance, case isolation, health education, environment disinfection and class/grade/school closure) and three pharmaceutical (symptomatic treatment, prophylaxis, vaccination) interventions. Symptom surveillance was implemented in 14 outbreaks. Case isolation, symptomatic treatment, health education and environment disinfection were employed in each outbreak. During four outbreaks (three H1N1pdm and one B subtype), class or grade closure was employed because cluster cases occurred in the class or grade, and school closure was employed in nine outbreaks (six H1N1pdm and three H1N1). Vaccination was employed in two outbreaks (one H1N1pdm and one H3N2). Prophylaxis was only employed in one H1N1 outbreak.

According to whether the intervention was employed or not, we divided the 15 outbreaks into employed groups and non-employed groups. Then we compared the mean $R$ of the two groups to infer if the intervention was 


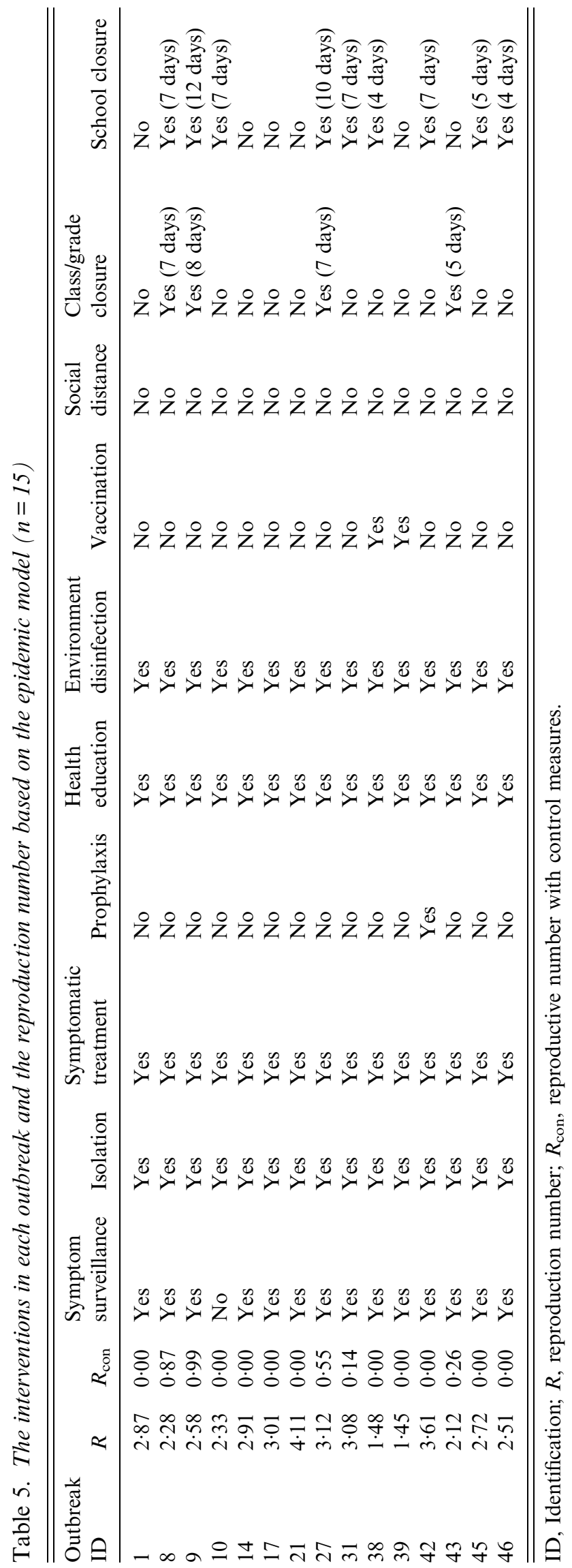

employed based on a high $R$. There was no significance of class/grade closure $(t=-0.492, P=0.631)$, school closure $(t=-0 \cdot 286, P=0 \cdot 780)$ or prophylaxis $(t=-1 \cdot 405, \quad P=0 \cdot 184)$. On the other hand, there was significance of vaccination $(t=3 \cdot 479, P=0 \cdot 004)$, but before the countermeasure was chosen, the $R$ of the employed group was lower than that of the non-employed group. These results reveal that the intervention was not employed based on whether the value of $R$ was high or not.

The reproductive number with control measures $\left(R_{\text {con }}\right)$ of each outbreak was calculated by the epidemic model using the reported data which was collected after the reported date. We observed that $R_{\text {con }}$ was $<1$ after the interventions were implemented and most $R_{\text {con }}$ were 0 (see Table 5 and Fig. 1).

\section{DISCUSSION}

Our results reveal that the mean value of $R$ in small-scale outbreaks was higher than in large-scale ones (outbreaks which occurred in a neighbourhood, city or province) in which $R$ was $<2 \cdot 0[2-5,9,13$, 14], whether or not $T c=2.7(t=3.068, P=0.007)$ or $T c=2.9(t=3.494, P=0.003)$, as calculated by the epidemic model. This implies that the attack rate and the peak incidence of a small-scale outbreak may be higher than those occurring in a city. The reasons for this difference are not clear, but may be caused by the high population density and contact frequency in a school or a prison compared to a neighbourhood. This may alert us to employ different countermeasures to control small-scale outbreaks rather than outbreaks occurring in a city at large.

Furthermore, the $R$ values estimated by normal distribution, delta distribution, and gamma distribution models were higher than those calculated by the epidemic model. The former three models resulted in a higher S.D. and larger ranges of $R$, which might make the estimation more discrete and unstable. We conclude that the former three models seem to more easily overestimate the $R$ of an influenza outbreak. And this overestimation may result in the control strategies being implemented excessively when the primary health department makes an emergency response policy. Thus, in the four models of the intrinsic growth rate method, the epidemic model might be the best one to recommend to estimate the transmissibility of influenza at the early stage of an outbreak.

We found that the commonly used interventions were symptom surveillance, case isolation, symptomatic 

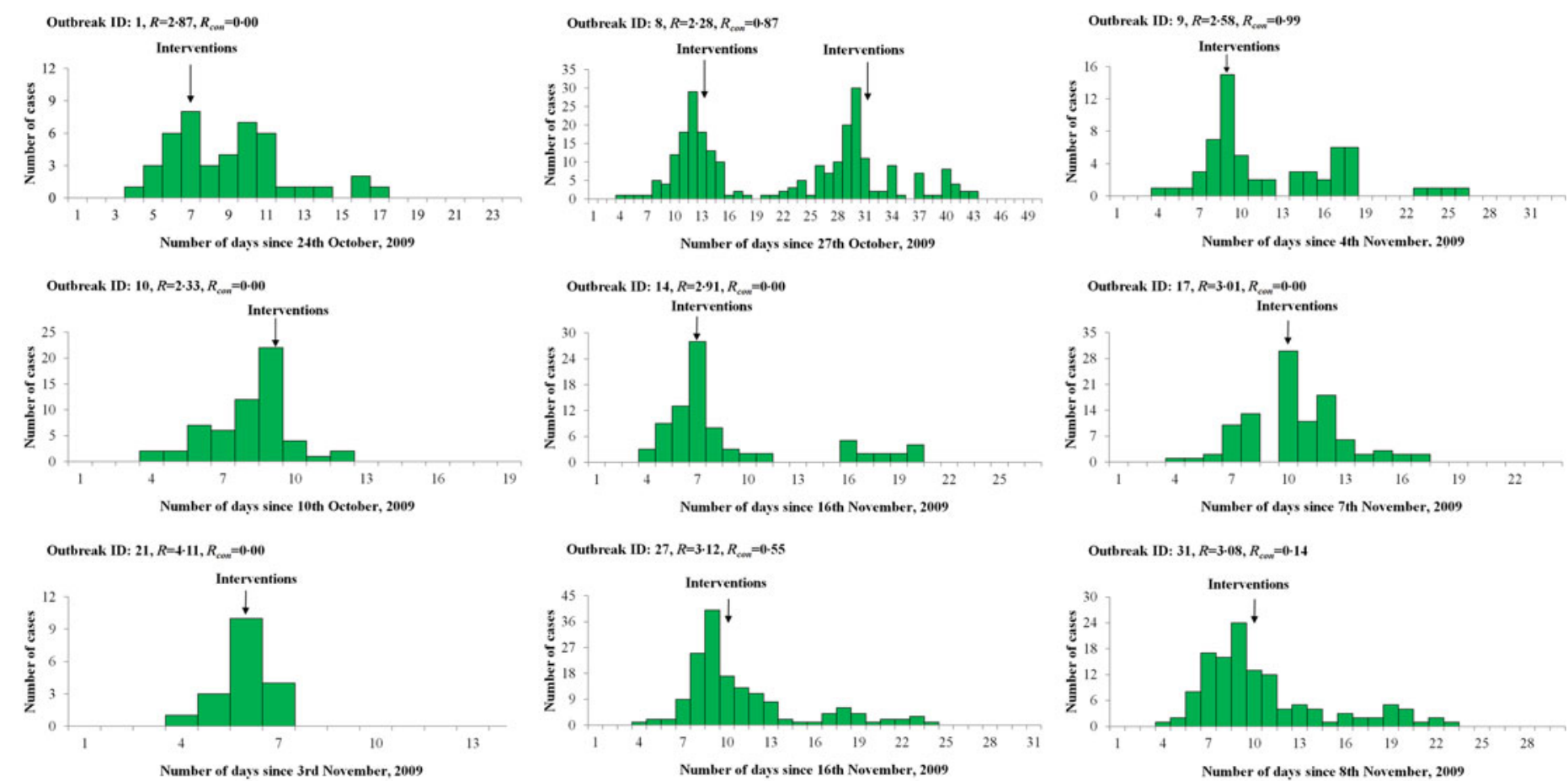

Outbreak ID: $38, R=1 \cdot 48, R_{\text {cen }}=0.00$

Outbreak ID: $39, R=1 \cdot 45, R_{\mathrm{con}}=0 \cdot-60$

Outbreak ID: $42, R=3-61, R_{\text {cont }}=0.00$
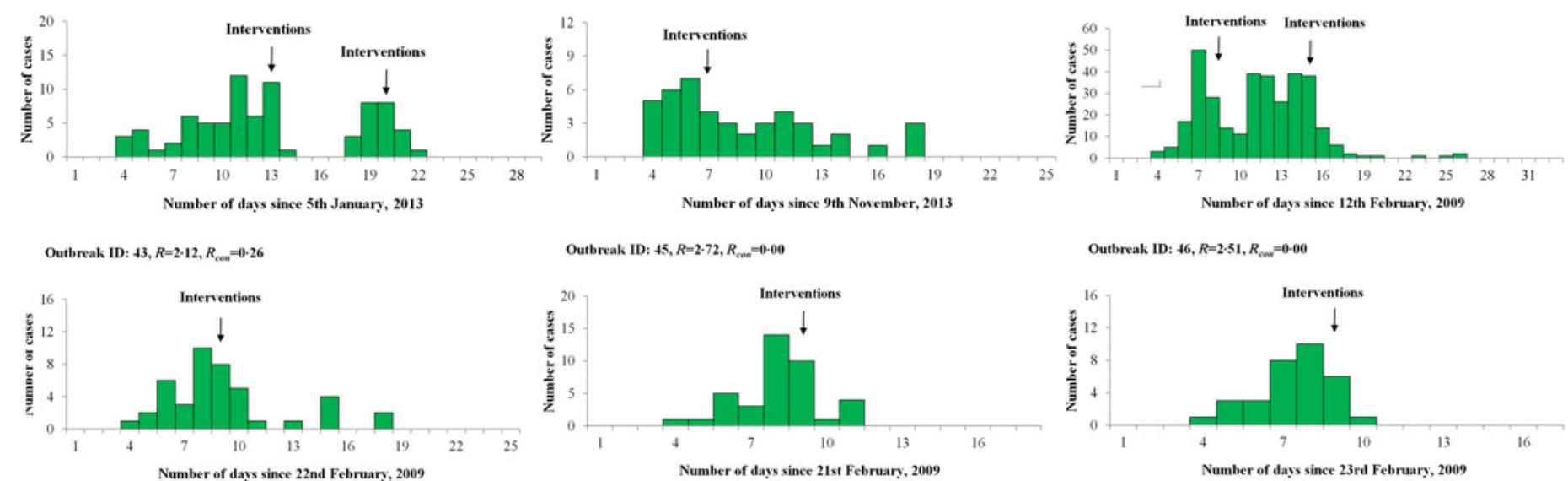

Fig. 1. Epidemic curves of 15 influenza outbreaks used for calculating $R$ based on an epidemic model in Changsha city, China. ID, Identification; $R$, reproduction number; $R_{\text {con }}$, reproductive number with control measures. 
treatment, health education and environment disinfection, followed by class/grade/school closure, vaccination and prophylaxis. They were not employed based on the transmissibility (the reproduction number $R$ ) of the outbreak. Although the transmissibility of most of the outbreaks decreased rapidly after the interventions, some of them were still close to 1 , which would prolong an outbreak. Therefore, we recommend that local CDCs calculate the reproduction number of the outbreak by using the epidemic model and the data when they receive an outbreak report, thereby avoiding implementing countermeasures blindly.

It should be noted that this study has some limitations because of the lack of integrity of the data and the methodology of the model. Regarding the data we collected, epidemic curves of seven outbreaks were missing or not integrated, and there were 19 outbreaks showing no exponential growth in early stages, which meant that there was still more effort required from the primary health department to improve the quality of the data collection. If the data is collected more accurately through field investigation, the $R$ estimation will be shown to be more precise. On the other hand, the epidemic model requires that the early stage of an outbreak must be $\geqslant 3$ days. The lower time span (especially $<3$ days) of the data may make the estimation unstable and unreliable. Finally, the epidemic model is only suitable to estimate the $R$ of the outbreak in the early stage according to the principle of the model, which signified that we could not use the whole data if the outbreak was reported after the early stage. Therefore, after the exponential growth stage, particularly for the stage after the epidemic peak of the outbreak, other more complicated models should be utilized, such as an ordinary differential equation model or a stochastic individual-based model to conduct the transmissibility estimation.

In conclusion, we have shown the mean value of $R$ of influenza outbreaks in Changsha, China, and our findings may show an appropriate example for the primary health department to assess the transmissibility of small-scale influenza outbreaks with a practical, yet accurate model at the early stage.

\section{ACKNOWLEDGEMENTS}

We thank Yelan Li and Zhi Xie from the Office for Disease Control and Emergency Response, Changsha Center for Disease Control and Prevention for helpful discussions. This work was supported by the National Natural Science Foundation of China (no. 81273192).

\section{DECLARATION OF INTEREST}

None.

\section{REFERENCES}

1. Chen T, et al. Risk of imported Ebola virus disease in China. Travel Medicine and Infectious Disease 2014; 12: 650-658.

2. Liu R, et al. The effectiveness of age-specific isolation policies on epidemics of influenza $\mathrm{A}(\mathrm{H} 1 \mathrm{~N} 1)$ in a large city in Central South China. PLoS ONE 2015; 10: $\mathrm{e} 0132588$.

3. Longini Jr. IM, et al. Containing pandemic influenza at the source. Science 2005; 309: 1083-1087.

4. Longini Jr. IM, et al. Containing pandemic influenza with antiviral agents. American Journal of Epidemiology 2004; 159: 623-633.

5. Yang Y, et al. The transmissibility and control of pandemic influenza $\mathrm{A}(\mathrm{H} 1 \mathrm{~N} 1)$ virus. Science 2009; 326: 729-733.

6. Chen SC, Liao CM. Modelling control measures to reduce the impact of pandemic influenza among schoolchildren. Epidemiology and Infection 2008; 136: 1035-1045.

7. Heffernan JM, Smith RJ, Wahl LM. Perspectives on the basic reproductive ratio. Journal of the Royal Society, Interface 2005; 2: 281-293.

8. Wallinga J, Lipsitch M. How generation intervals shape the relationship between growth rates and reproductive numbers. Proceedings of the Royal Society of London, Series B: Biological Sciences 2007; 274: 599-604.

9. Trichereau J, et al. Estimation of the reproductive number for $\mathrm{A}(\mathrm{H} 1 \mathrm{~N} 1) \mathrm{pdm} 09$ influenza among the French armed forces, September 2009-March 2010. Journal of Infection 2012; 64: 628-630.

10. McBryde E, et al. Early transmission characteristics of influenza $\mathrm{A}(\mathrm{H} 1 \mathrm{~N} 1) \mathrm{v}$ in Australia: Victorian state, 16 May-3 June 2009. Eurosurveillance 2009; 14: pii:= 19363.

11. Hahné S, et al. Epidemiology and control of influenza A $(\mathrm{H} 1 \mathrm{~N} 1) \mathrm{v}$ in the Netherlands: the first 115 cases. Eurosurveillance 2009; 1: pii: $=19267$.

12. Chen T, et al. Investigation of key interventions for shigellosis outbreak control in China. PLoS ONE 2014; 9: e95006.

13. Germann TC, et al. Mitigation strategies for pandemic influenza in the United States. Proceeding of the National Academy of Sciences USA 2006; 103: 59355940.

14. Tracht SM, Valle SYD, Hyman JM. Mathematical modeling of the effectiveness of facemasks in reducing the spread of novel influenza A(H1N1). PLoS ONE 2010; 5: e9018. 